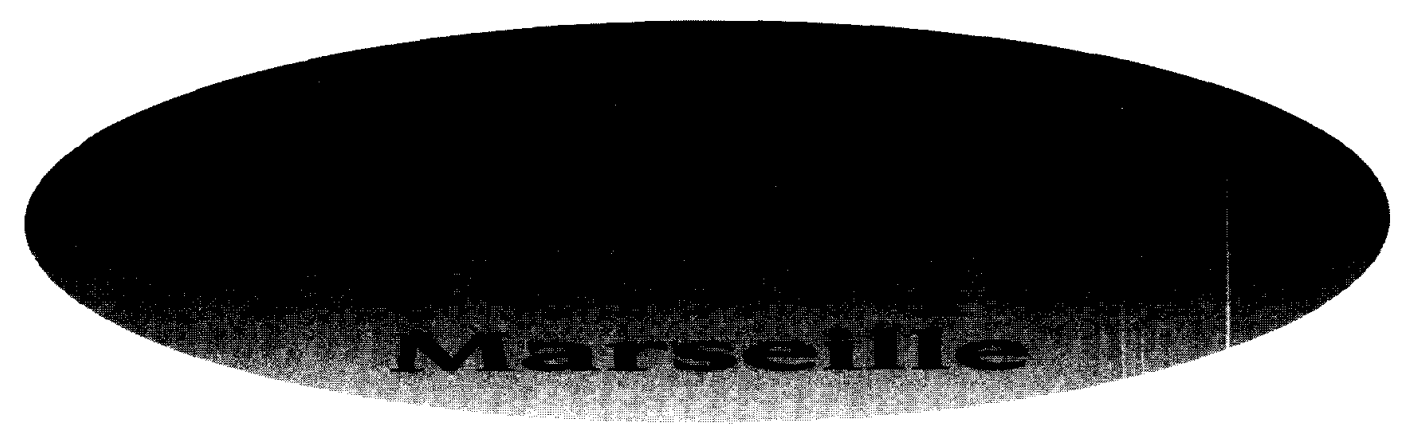

\title{
SINGLE TOP PRODUCTION AT LEP II
}

\author{
O. Panella $^{a}$, G. Pancheri $^{b}$, And Y. N. Srivastava $^{c}$ \\ ${ }^{a}$ Centre de Physique Théorique, CNRS Luminy \\ Case-907, F-13288 Marseille, France \\ ${ }^{b}$ Laboratori Nazionali di Frascati dell'INFN, Frascati, I-00044, Italy \\ ${ }^{c}$ Dipartimento di Fisica and INFN, Universitá di Perugia, Italy
}

\begin{abstract}
We study the production of a single top quark through the processes $e^{+} \gamma \rightarrow$ $\nu t \bar{b}, e^{+} e^{-} \rightarrow W^{*} \gamma^{*} \rightarrow t \bar{b}+e \nu$, and $e^{+} e^{-} \rightarrow W^{*} W \rightarrow t \bar{b}+e \nu$ within the minimal Standard Model. Numerical estimates of the signal cross-sections at LEP II energies are compared with pair production below threshold, $e^{+} e^{-} \rightarrow$ $t \bar{t}^{*} \rightarrow t W^{*} \bar{b} \rightarrow t \bar{b}+e \nu$. We show that if $m_{t o p}>\sqrt{s} / 2$, the $W W^{*}$ and $W^{*} \gamma^{*}$ channels are dominant, but these processes give an interesting event rate only if the expected luminosity could be ameliorated relative to present estimates.
\end{abstract}

September 1993

CPT-93/P.2937

CNRS Luminy - Case 907 - CPT - F 13288 Marseille Cedex 9 - France

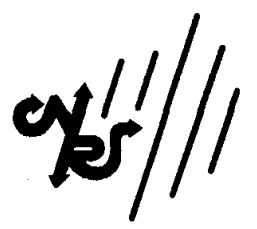



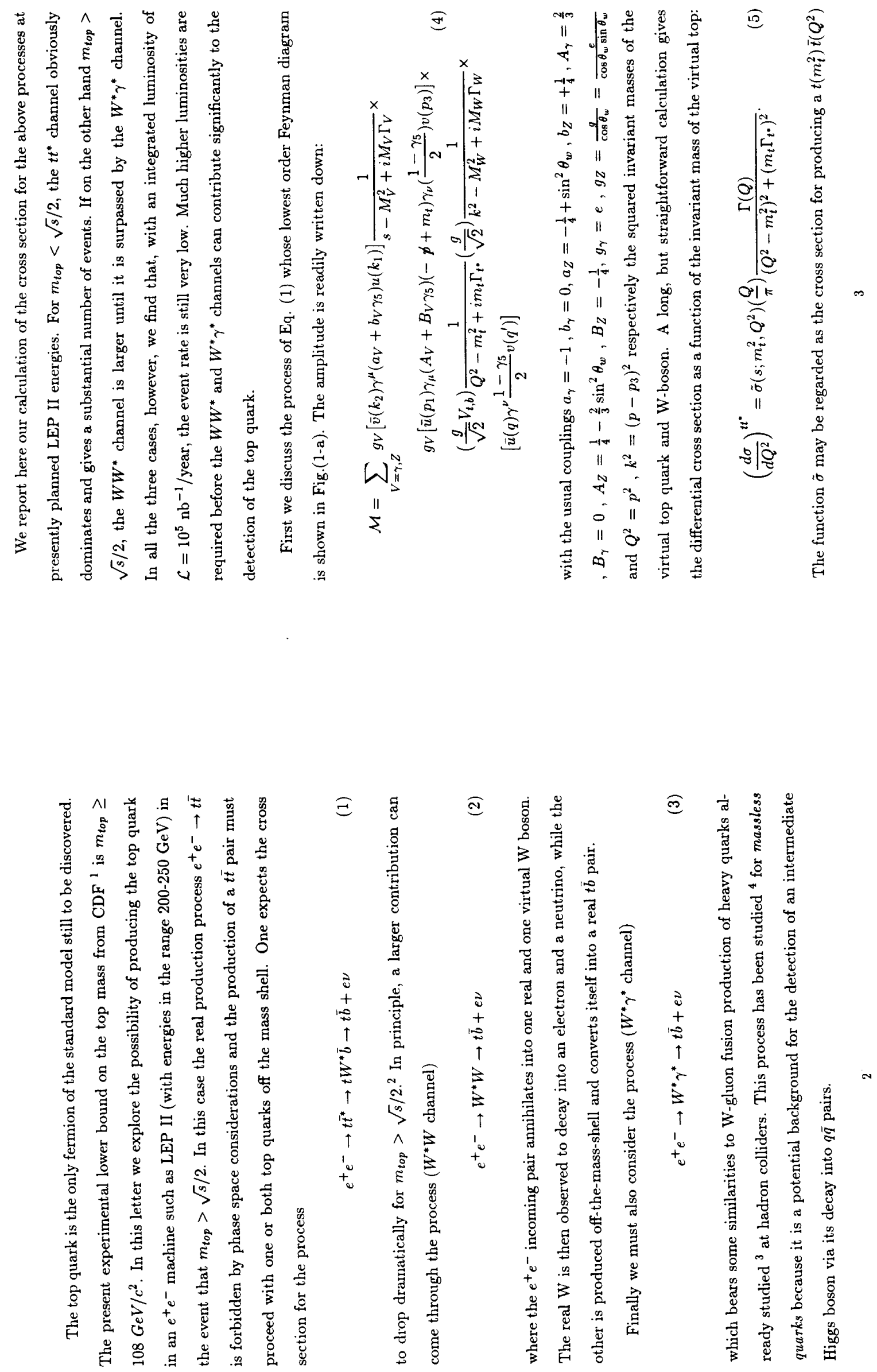

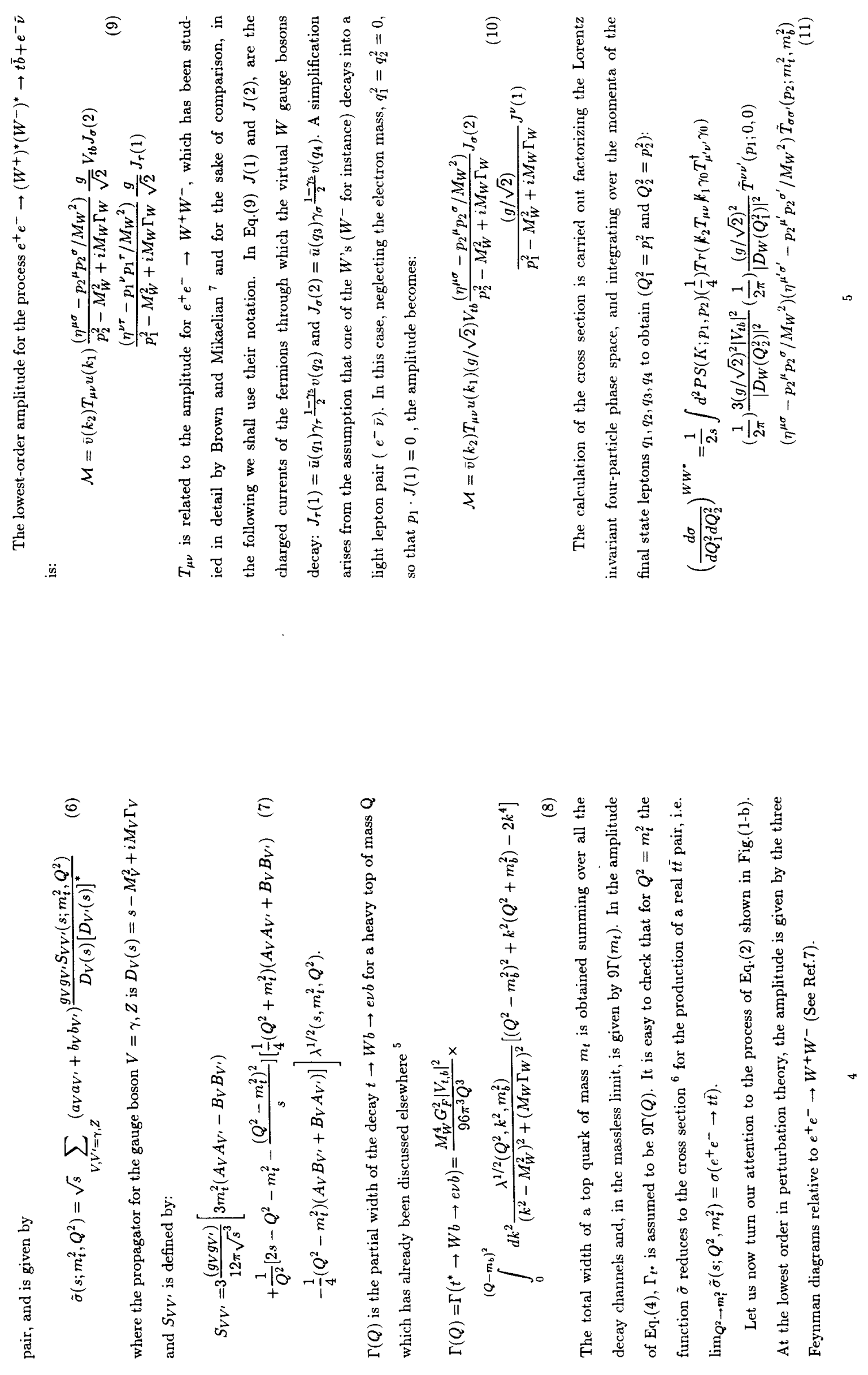

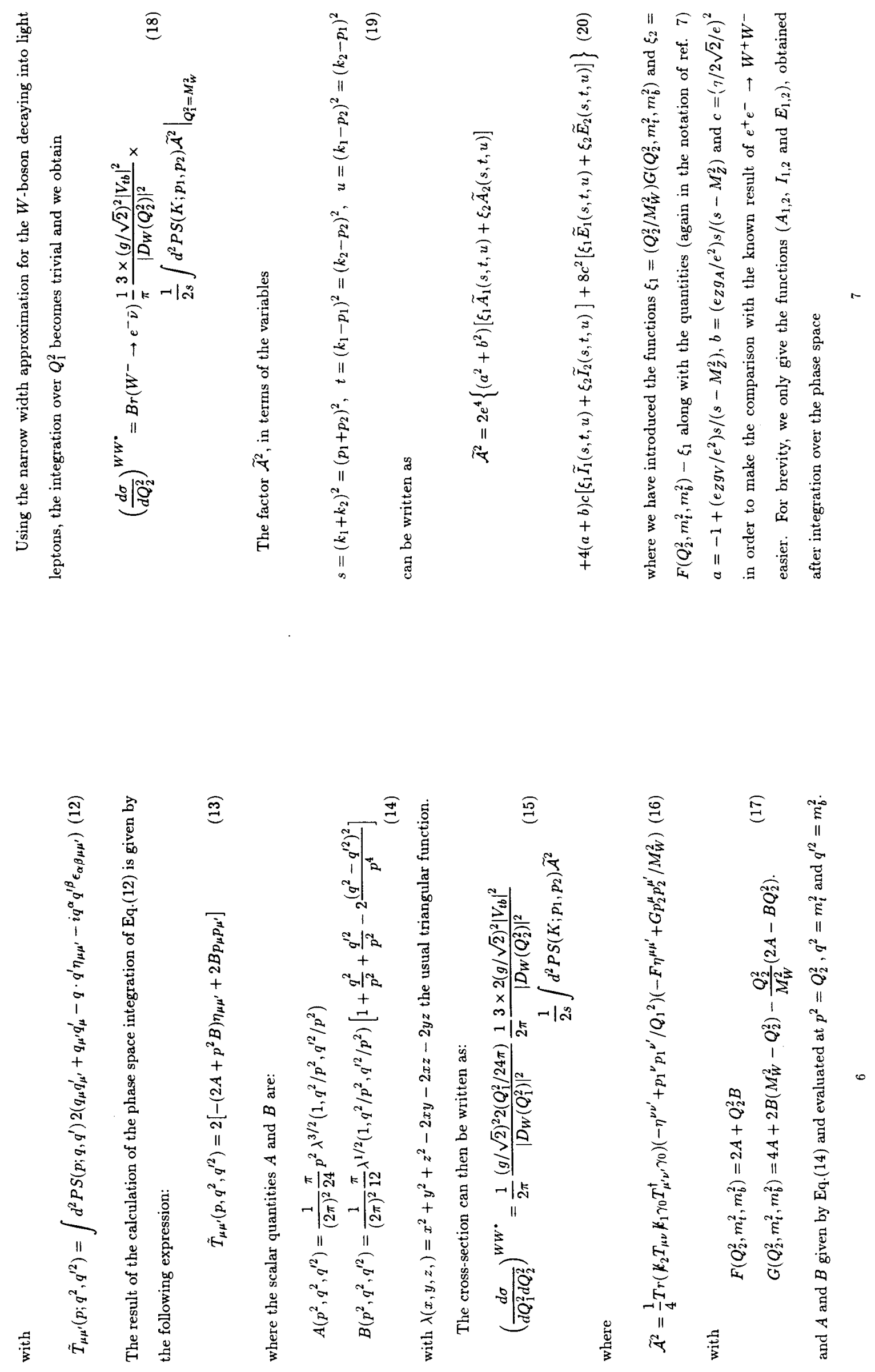


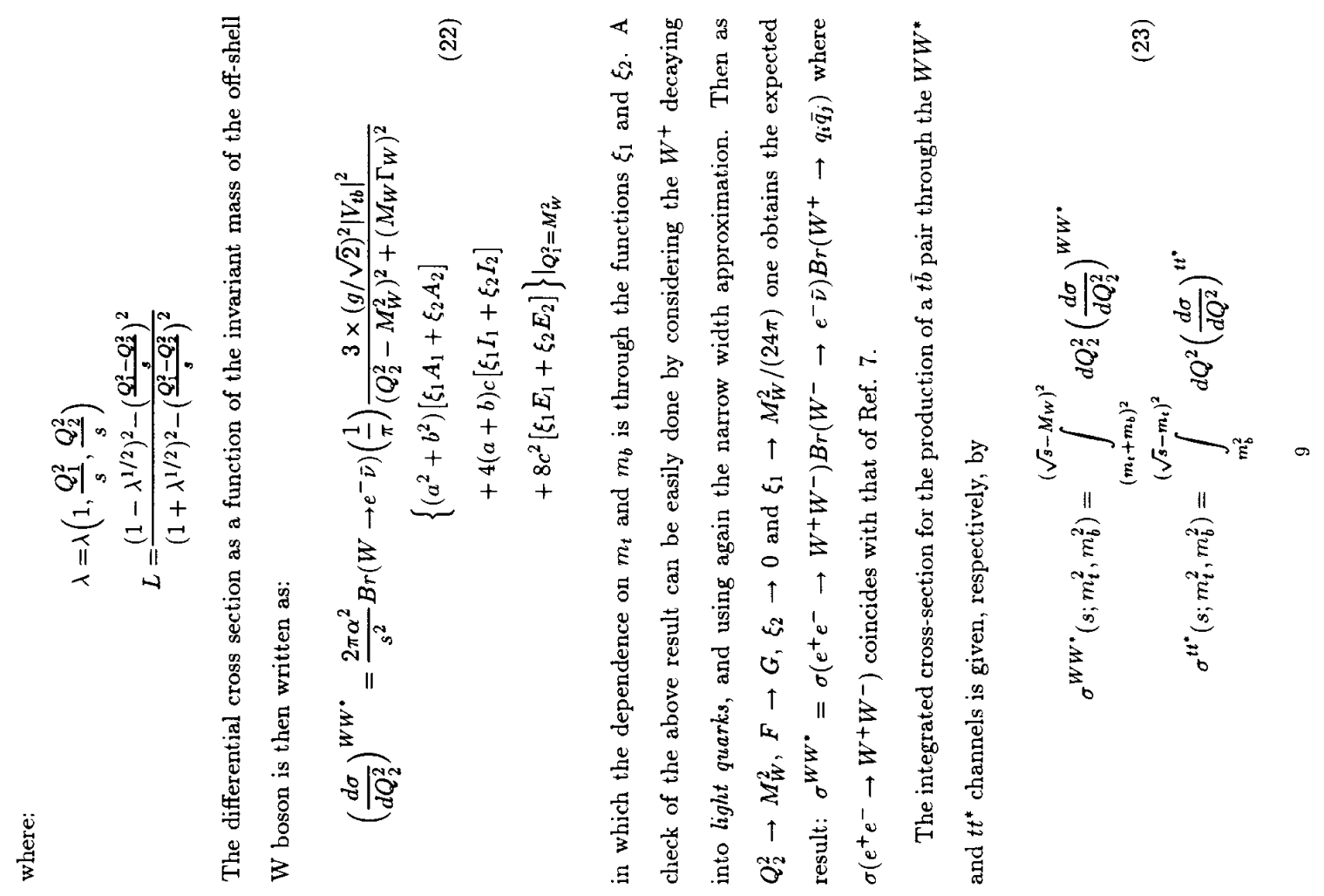

ลิ

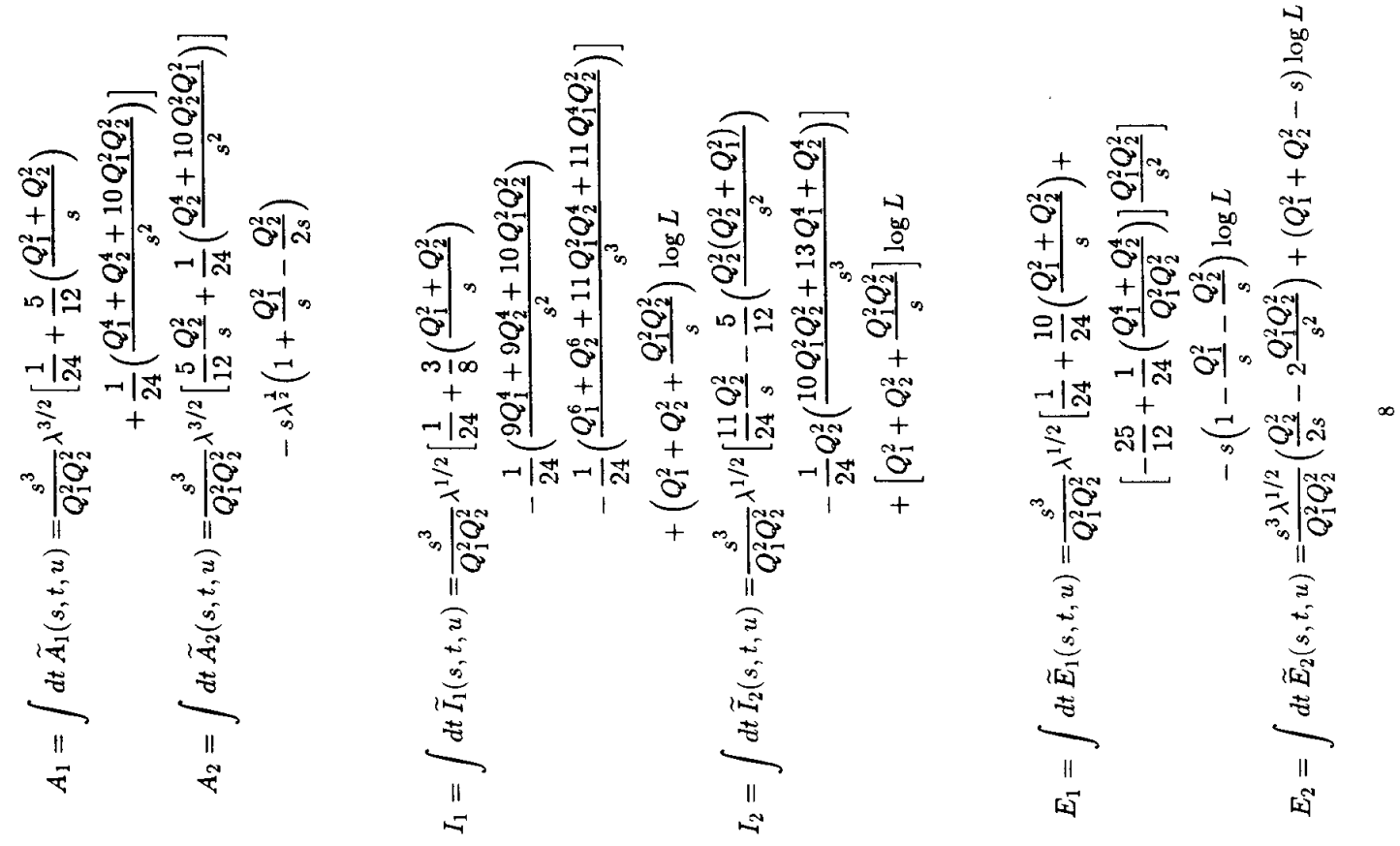




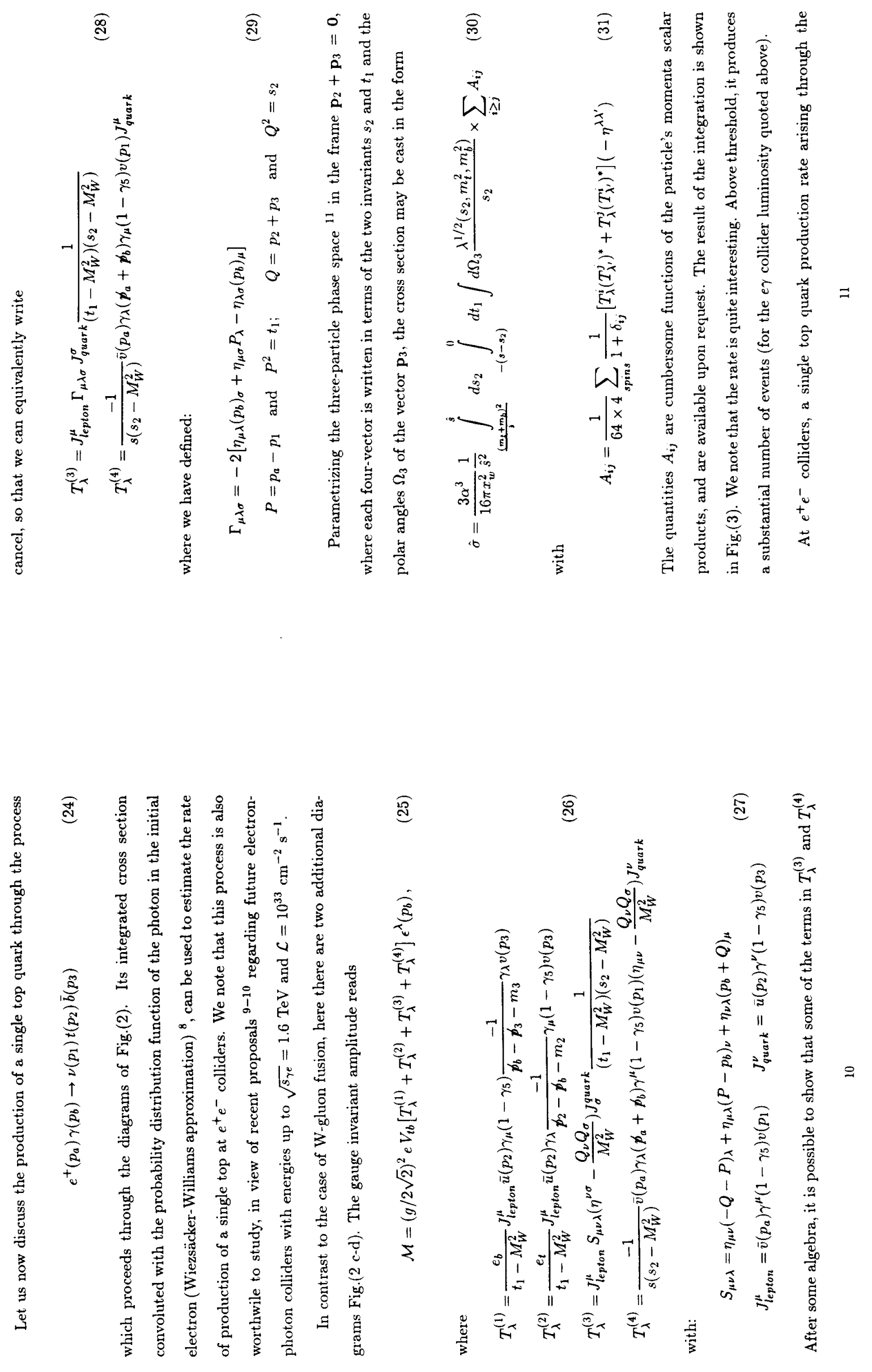




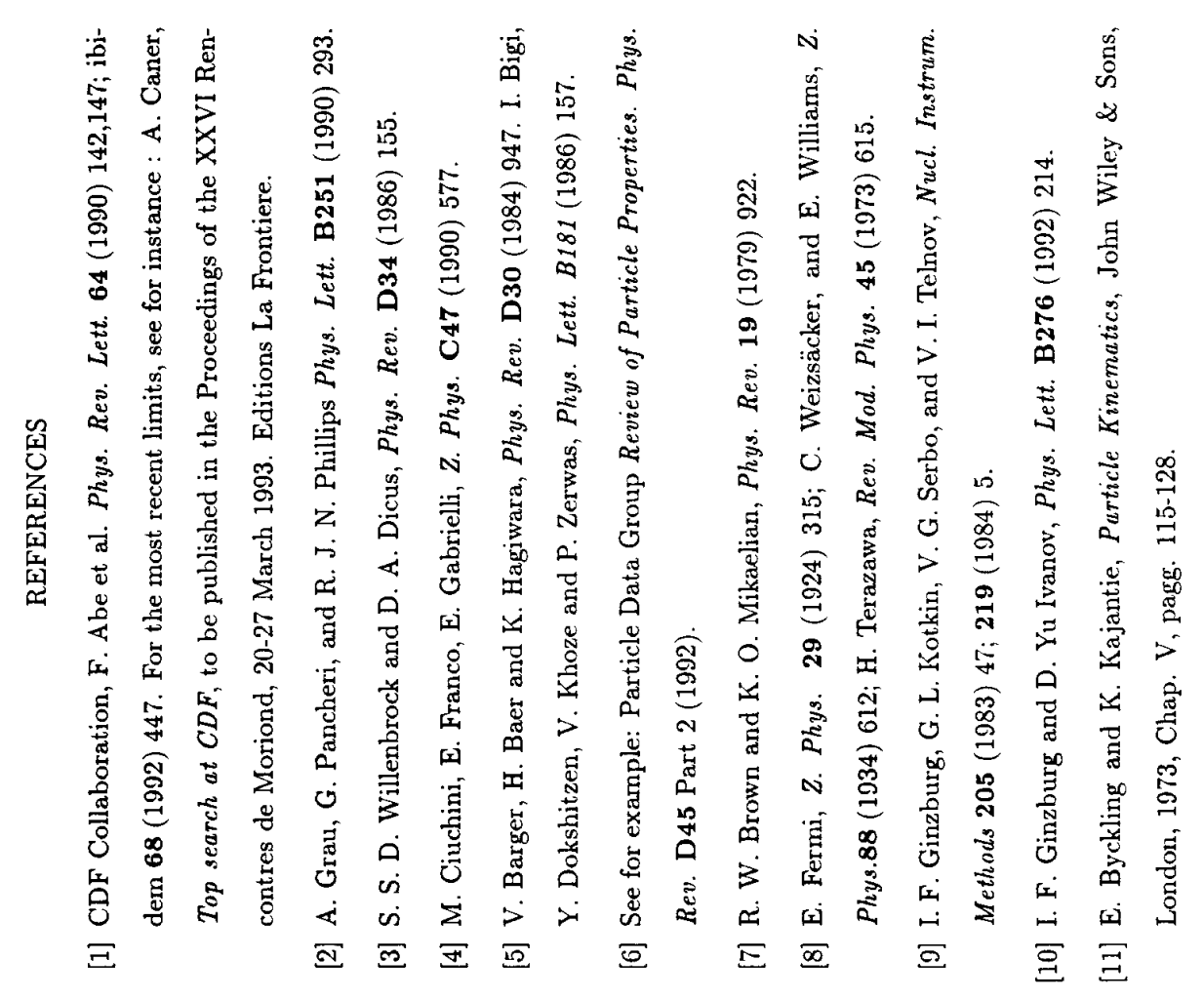

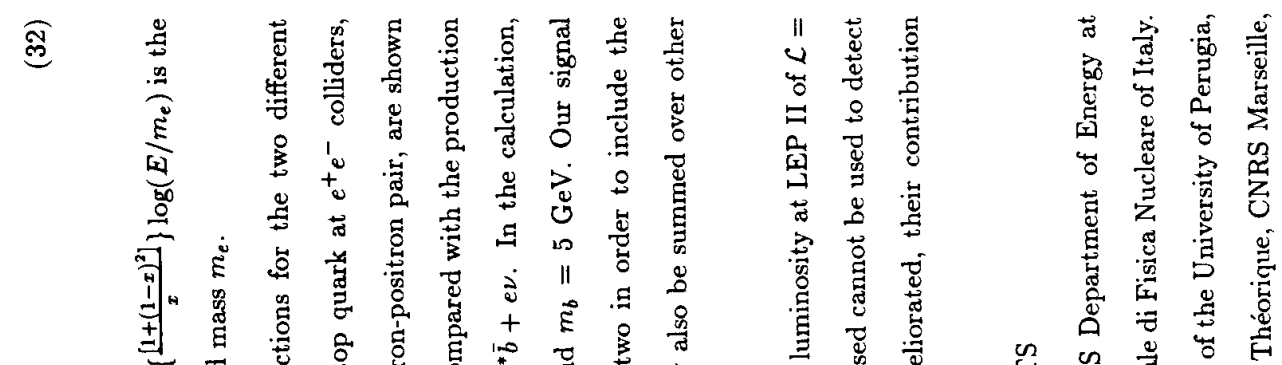

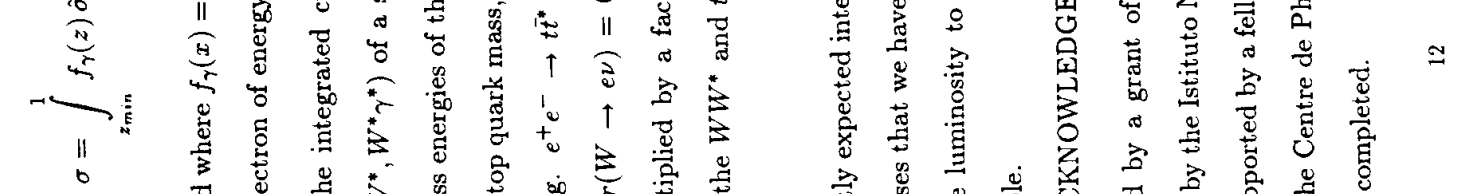

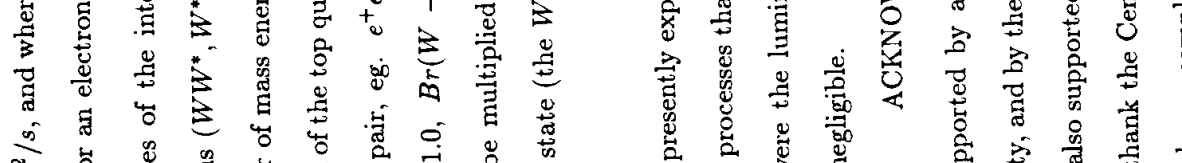

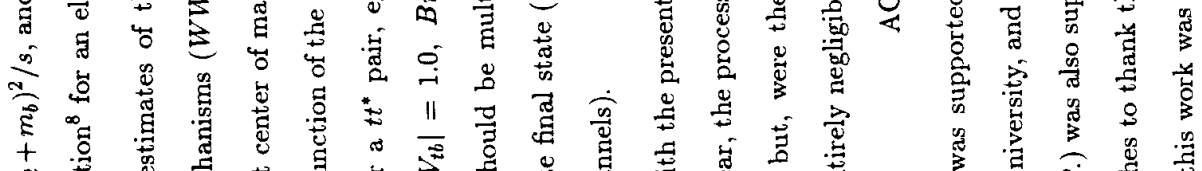

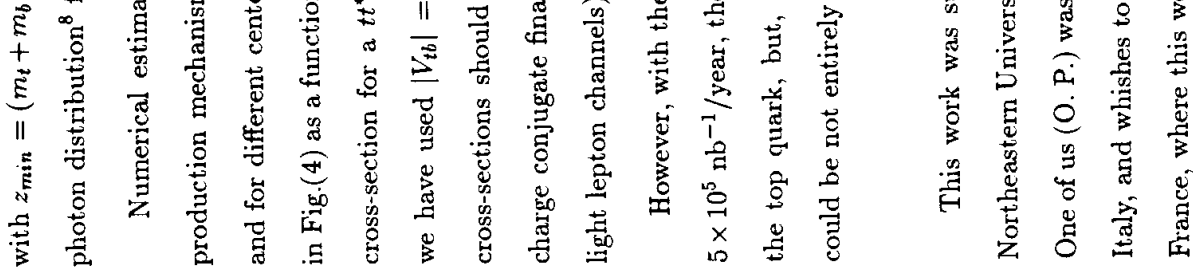



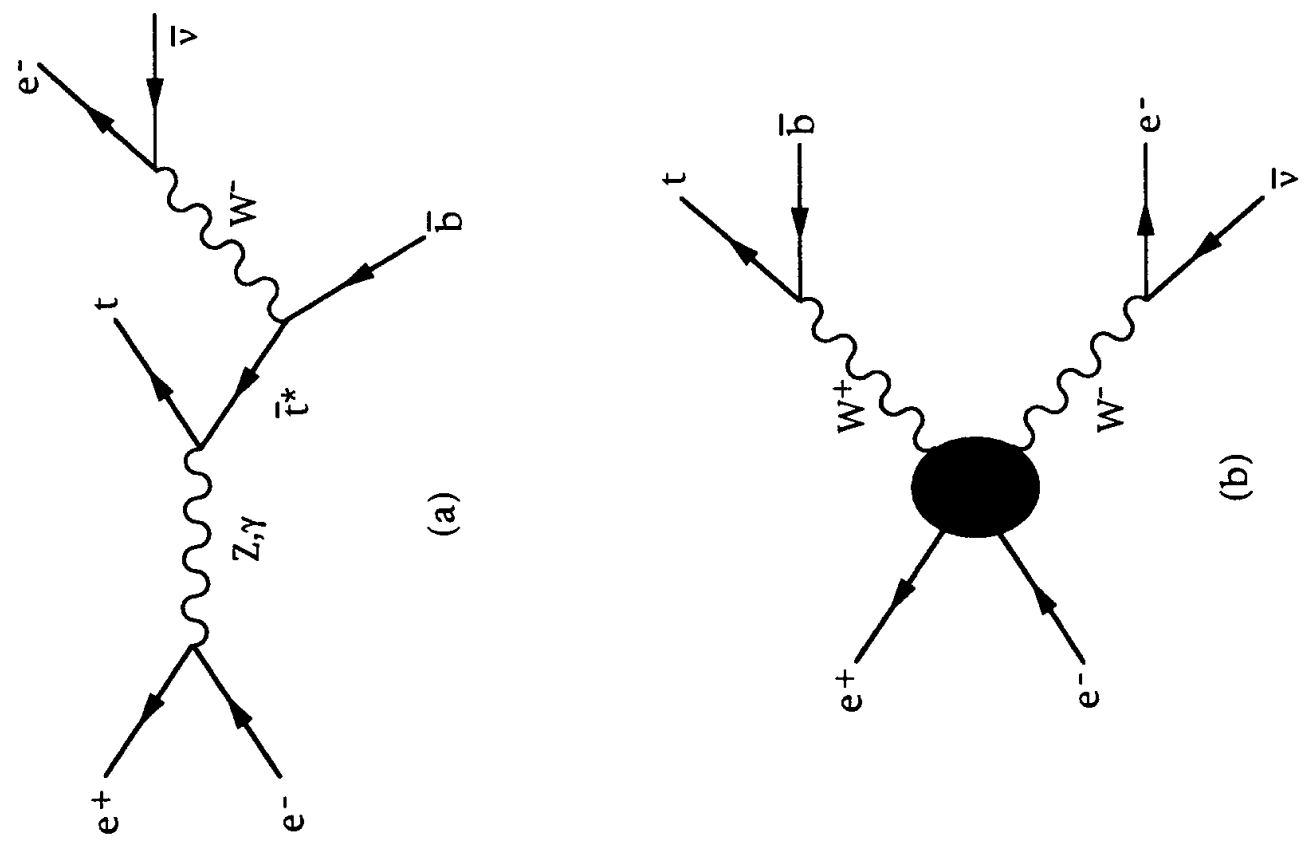

电

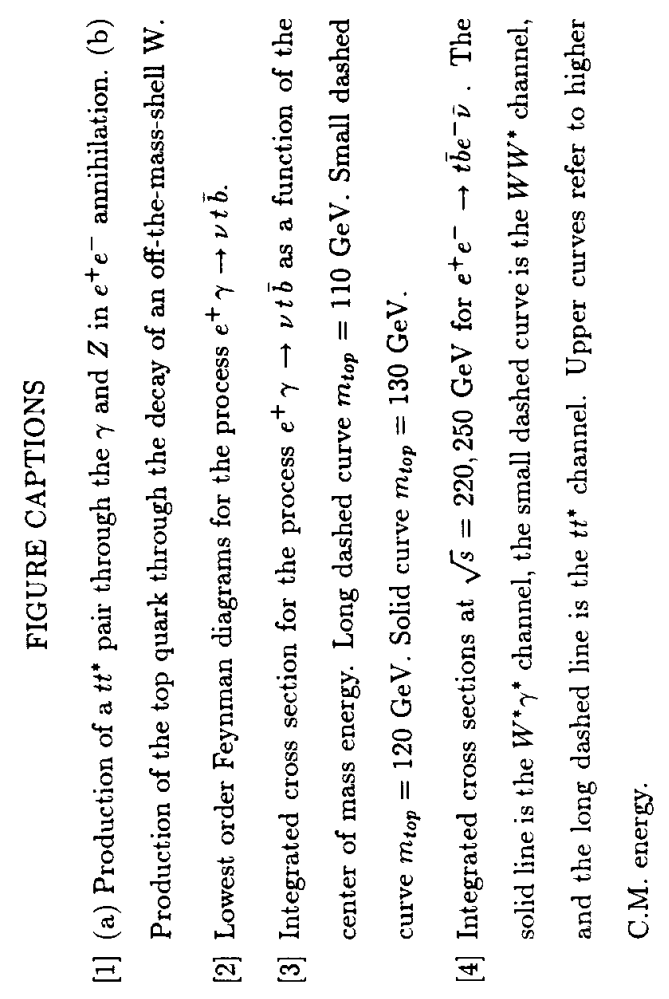




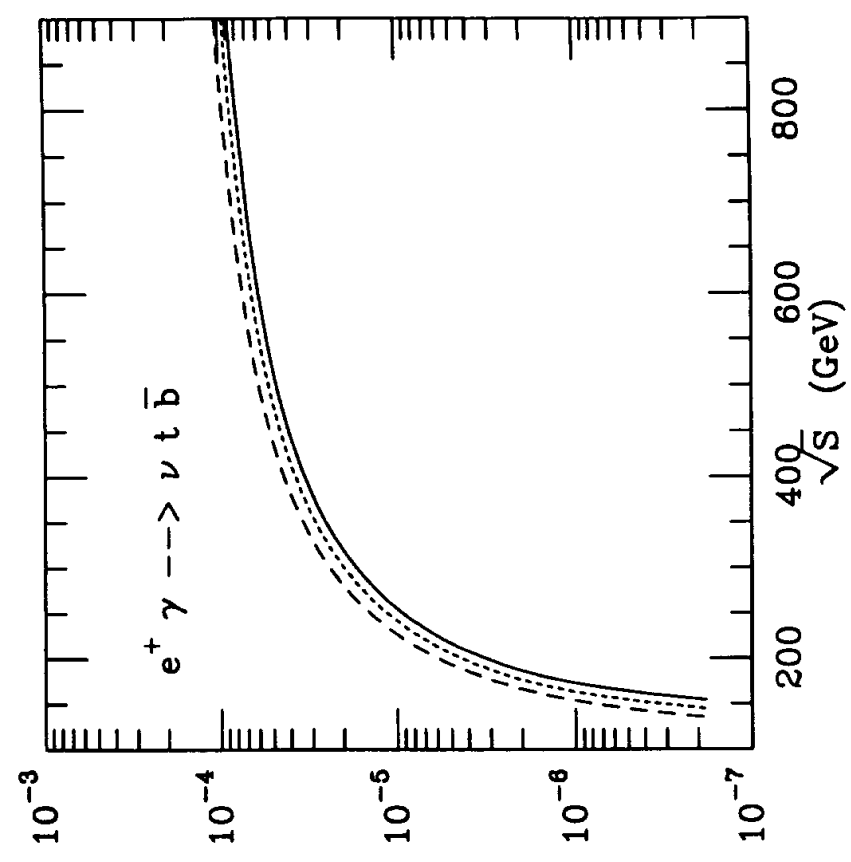

(qu) 0
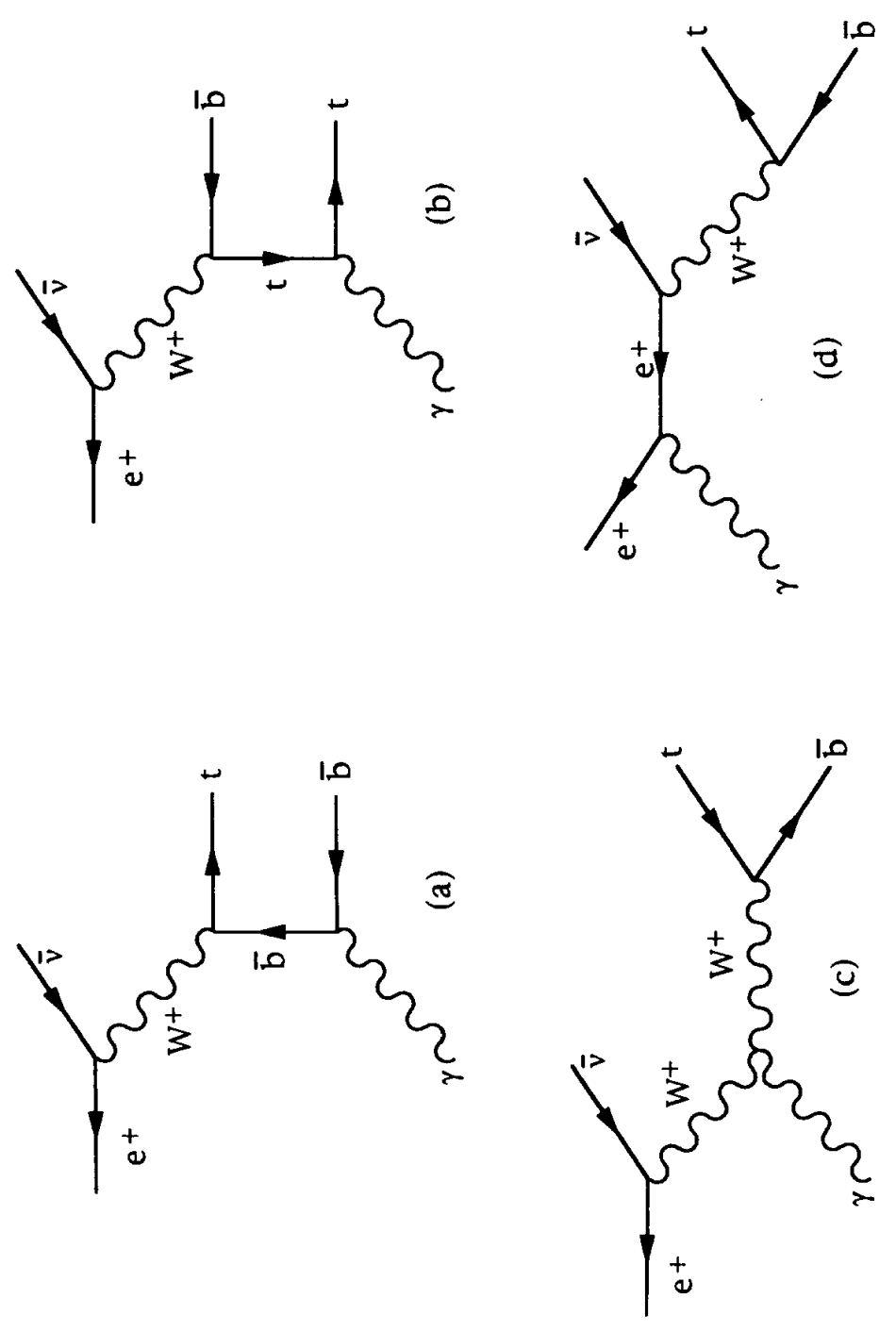


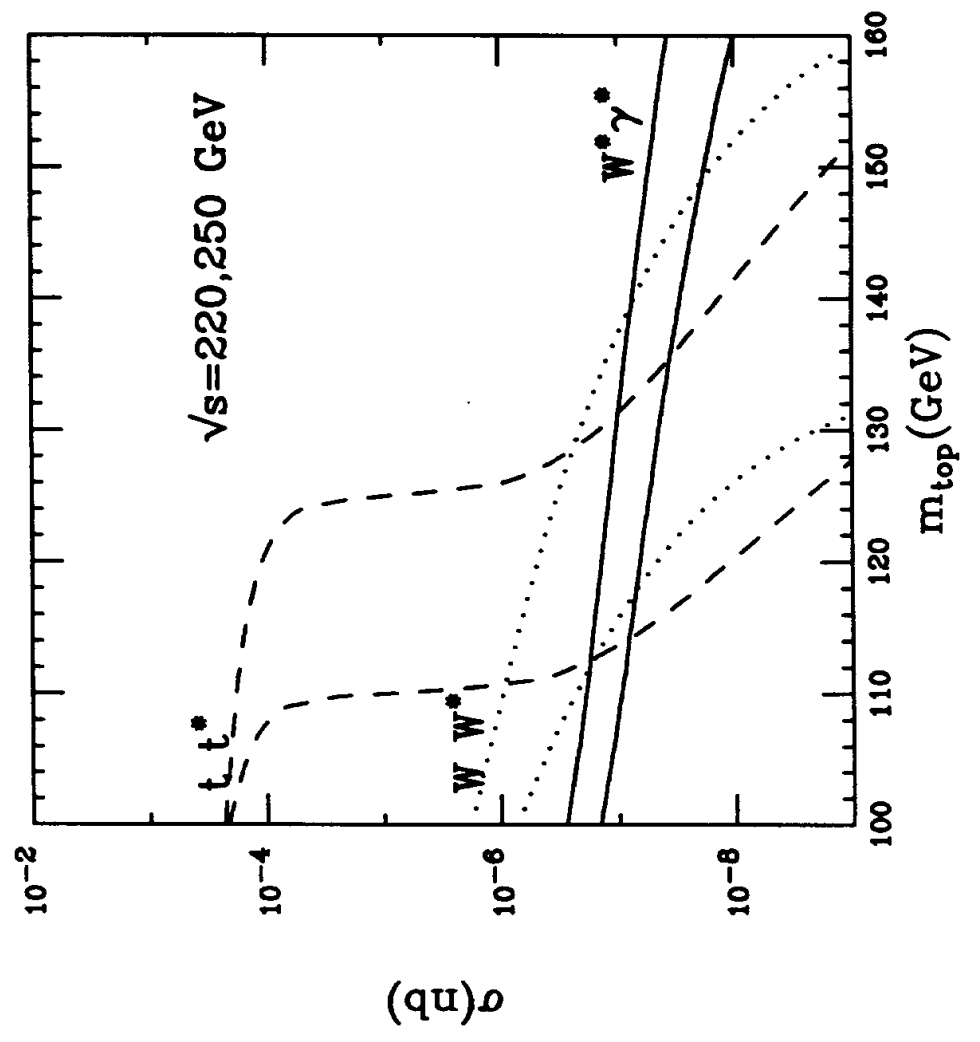

量 
